\title{
IMPROVING THE USER EXPERIENCE IN THE FIELD OF EHEALTH. MUOVITI! A CO-DESIGNED TRANSMEDIA APPLICATION
}

\author{
Letizia Bollini and Monica Pennati \\ University of Milano-Bicocca, Piazza dell'Ateneo Nuovo 1, 20126 Milano, Italy
}

\begin{abstract}
The paper presents and discusses a participatory project of a transmedia application Muoviti! based on biofeedback data to be used both with smartphones and wearable devices aimed to help people practising a regular and moderate physical activity in order to stay healthy and motivated. The application has been co-design with users - both female and male, age 30-50 - using a participatory approach to understand the needs, aspirations and behaviours of this particular segment of people. Different human-centered design research methods have been adopted - such as co-design workshops, personas $\&$ scenarios based user tests, open questions interviews, and surveys - to have deep insights according to different criteria included in the Behavioral Change Techniques taxonomy developed by Michie. The results of the experimental phase - along with an extensive benchmark focused on commercial applications already shared on the mobile marketplaces, and an expert heuristic analysis - have suggested significant changes in the app's features, user experience, and interface design, which have been assessed with users in a second design/user test iterative cycle. In particular, the people feedbacks and dislikes were addressed to the competition culture, and privacy issues. In a further stage of the research project, a pilot of the software will be tested by the users after a month-long use to evaluate the impact and effectiveness of the app in the health behaviour-changing process.
\end{abstract}

\section{KEYWORDS}

Mobile App User Experience Design, Ehealth, Co-design, Transmedia Application Design, Mobile User Interface Design

\section{INTRODUCTION}

Man was born to move (Alessandri and Viroli, 2007, pp. IX) but when this does not happen, people wellness is at risk. Indeed, sedentary lifestyle has been declared the disease of our century: it is the fourth risk factor of chronic non-communicable diseases and one of the primary causes of death in the world. On the other hand, physical activity promotes well-being reducing the incidence of some disease (i.e. heart disease, hypertension, cholesterol) and improves mental performance and mood. It is therefore imperative to promote physical activity, especially among people over forty, who face profound physiological changes. Unfortunately, prevention policies still not to be effective and there are few investments in this area. Nevertheless, information technologies could increase the success of interventions.

Thanks to a continuous and ubiquitous interaction digital devices engage users and identify their performance trends: based on user's sociodemographic and psychological data, behavioural data (quantity and type of p.a) and contextual information (Klein et al., 2015) these devices propose personalised exercises able to enhance lifestyle changes. These measures are composed of one or more Behavior Change Techniques (BCTs). Moreover, thanks to the support of a consistent data communication, wellness apps allow the user's self-monitoring (habits, efforts and progress) which feeds the self-efficacy perception (French et al., 2014), that induces and controls actions. The positive correlation between self-efficacy and an increasing and sustained p.a among adults has been demonstrated (Caulfield \& Donnelly, 2013; Kaewthummanukul \& Brown, 2006; Bauman et al., 2012; Ashford, Edmunds \& French, 2010; Burke et al., 2008). The execution of a target-behaviour conveys the perception of self-efficacy, while contrarily the failure decreases it. When people perform tasks and activities, they read their actions results by developing beliefs about their capabilities, so that they can act by these opinions. Based on these factors, a research project has been established (Baretta et al., 2016) to create an app aimed to encourage people's physical practice. 


\section{MUOVITI! A TRANSMEDIA MEDIA APP IN THE FIELD OF EHEALTH}

Muoviti! [Move yourself!] is a transmedia wellness app for smartphones and wearable devices direct to healthy people who are proposed to practice sport as a primary prevention measure. The purpose of the app is to encourage user and support a fairly intensive workout in order to receive all the self-protective mental and body benefits. The app is based on heart-rate monitoring during cardio-training such as running, walking or bicycling. The users' heartbeat is recorded to alert him/her in real time when under the aerobic threshold to boost the intensity of the activity and return to a suitable level.

Based on WHO guidelines (2010), cardiac stress must be maintained for at least 10 minutes in order improve health like the improvement of aerobic capacity, exercise tolerance, greater muscle oxygenation, lower heart rate and blood pressure, the decrease in triglycerides in the blood and the increase of the good one. In particular, the target group (30-50 years old, both male and female subjects) should perform moderate physical activity (60-70\% of maximum heart rate) for 150 minutes or intense activity (70-80\%) for 75 minutes per week, corresponding to a quota of 600 MET (WHO, 2010; Haskell et al., 2007). The innovation stands in the software ability to combine physiological and behavioural data with psychological ones to suggest personalised goals. From the intersection of physical activity data and self-efficacy perceived by the user (psychological variable), the model can automatically generate a goal to be achieved, which is redefined weekly based on the user's performance and collected data. The physiological and behavioural variable will be extrapolated through the heart rate monitor, while the self-efficacy data will be entered by the user. At the end of each training, users will be asked to evaluate their ability to replicate a similar workout (duration and intensity) to the one just completed.

Physiological data are daily recorded by a PulseOn, a wearable heart rate monitor. Its wireless technology uses optical sensors to reveal blood flow below the skin: infrareds illuminate skin and capillaries while a photodetector catches backscattered light from the tissues. Blood flow changes according to the heart pumping frequency and PulseOn sensors detect this variation and process data to calculate heart rate (PulseOn, 2016). Thanks to the heartbeat data it is possible to determine the Physical Activity Energy Expenditure (PAEE) and consequently, the intensity of the physical activity performed, which is calculated in MET, the metabolic equivalent (Strath et al., 2013). Psychological data, on the other hand, are introduced into the application by the users, who are asked to evaluate the self-efficacy perceived. If the physical activity performed during the week turns out to be greater than or equal to the one target established, then the goal is considered achieved. Based on this data and self-efficacy, the target for the following week will be established (week $n+1$ ): if the level of self-efficacy of the user is high, the physical activity to be performed in the next week will be increased (increasing PA); if it is low, it will be the same as the previous week (PA maintenance). The ultimate goal of the application is to ensure that the users gradually increase (and at their own pace) his physical activity, up to 600 MET or 150 minutes of moderate activity (Haskell et al., 2007; WHO, 2010). In particular, Muoviti! considers only moderate or intense physical activity, not the light ones. Although the practice of the latter brings energy expenditure, it does not adequately stimulate the cardiovascular system to get the benefits previously listed. Also, the app will not allow the elimination of the workouts from the proposed plan nor the modification of physical activity amount to be performed. Like many applications, Muoviti! implements some features related to behaviour change techniques, to increase the effectiveness of the interventions. These functionalities were identified thanks to a benchmark and a co-design workshop.

\section{CO-DESIGNING WITH USERS: PARTICIPATORY METHODS}

In specific, the participatory activities have been the key elements of the whole design process. To better understand users needs and context, different kind of co-design workshops have been organised to elicit and assess expectations, needs, fears of people belonging to the target group. The choice to use participatory methods has been guided both by the desire to let the users select the features (innovative or otherwise) to be implemented in the app and by having more in-depth knowledge and understanding of the people behaviours. This practice of design collaboration (Rizzo, 2009) helped to combine visions, inputs and skills of different people in order to solve the given problem. The user assumes the role of co-creator while the designers/researchers play the role of facilitators. The latter, in fact, should interact with the users getting in tune with them, supporting their creativity and preventing them from falling into frustration (Bollini et al., 2014, Bollini, 2012a, 2012b). 


\subsection{The Co-Design Workshops}

The workshop sessions took place in July 2016 at the FIRB laboratory (see figure 1). They were organized to identify which tasks will be implemented in the app, during the session the subjects shared their sports habits and identified their ideal fitness app, how it should work and look like. Subjects, both men and women, age 30-50, took part to the co-design activities: characterized by quite different lifestyles and professions, they were chosen above all for their tendency to sedentariness and the difficult to do sport.

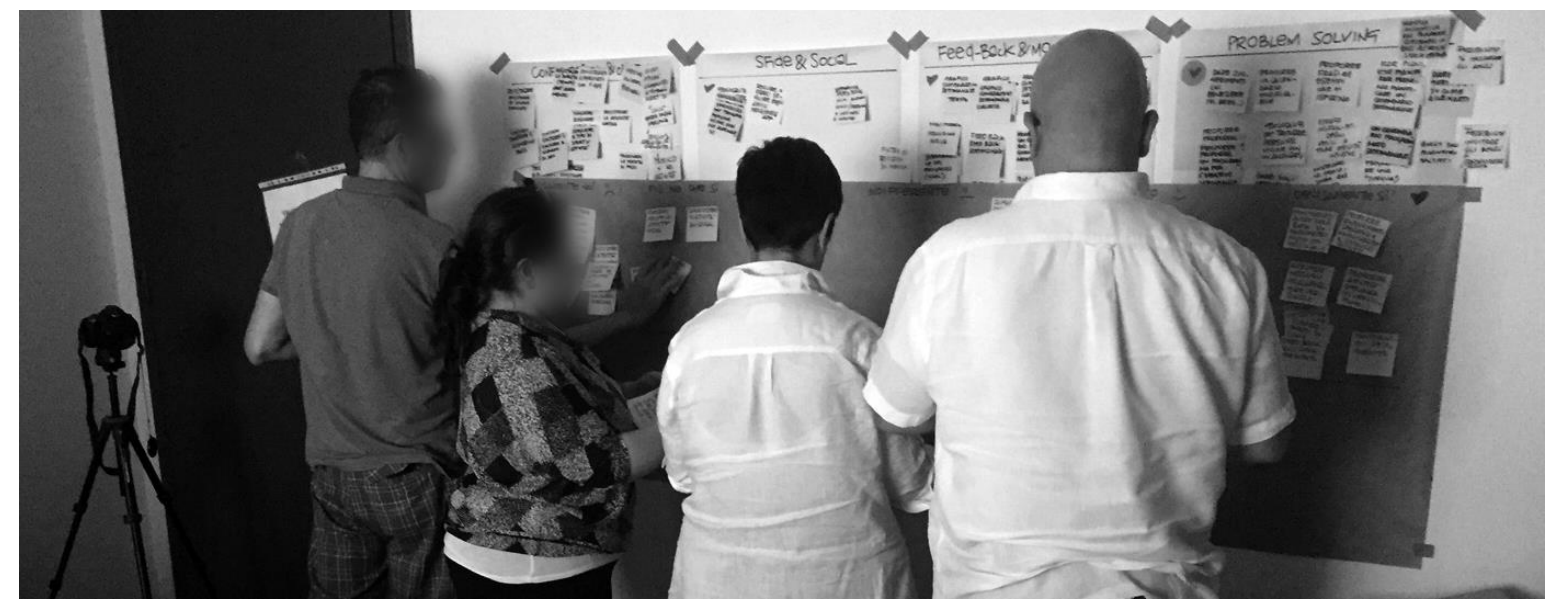

Figure 1. People during the co-design workshop in 2016: selecting, clustering and ranking the proposed app's features in the Semantic differential scale activity

Semantic differential scale questions together with basis workshops tools and other metaphorical materials previously prepared to solicit the discussion were used. Thanks to the benchmark and the questionnaire developed by Dario Beretta (2017), a serie of functionalities have been brought up to be implemented in Muoviti! to support the users' physical activity. The requirements were organized according to the benchmark categories, even though not all the functionalities found were re-proposed.

Three different typologies of tasks have been introduced and reviewed:

1) Feed-back and monitoring (11 features) - such as weekly life-circumference /weight gain or loss/calories consumption comparison chart and statistics (see fig. 2)

2) Configuration and objectives (11 features) - i.e. record the average speed, GPS route records, alert for entering data (see fig. 3)

3) Challenges and Social (10 features) - publish the training session on social media, geolocation to find nearby people to train with, launch a challenge to friends of the app, create and update the friends' ranking, just to mention the most important. (see fig. 4).

In particular, in the following category, functionalities are linked to one or more Behavioral Change Techniques belonging to Michie et al. taxonomy (2013).

4) Motivation (17) (see fig. 5)

- Keep track of improvements: Monitoring of behavior/Focus on past success)

- Monitor and receive feedback on some parameters: Monitoring/Feedback of outcomes of behavior

- Feedback on weight loss: Feedback on the outcomes of behaviour

- Propose specific activities for the improvement of physical appearance: Goal setting/outcomes

- Propose specific work to improve fitness levels: Goal setting - outcomes

- Monitoring of performance: Monitoring of behavior

- Receive feedback on the performances performed: Feedback on behavior

- Create groups to perform physical activity: Social support - emotional

- Monitoring of weight and life cycle: Monitoring of the outcome of behavior

- Track improvement and fitness indicators: Monitoring of the outcome of behavior/Focus on past success

- Receive messages from a personal trainer: Social support (practical)/Instruction on how to perform the behavior/Verbal persuasion about capability 
- Suggestions on what to do to lose weight: Instruction on how to perform the behaviour

- Defining challenging and set physical activity goals: Goal setting -Behaviour

- Win prizes for achieving goals: Non-specific reward

- Share physical activity on social networks: Social comparison

- Compare your performance with others: Social comparison

- Organize challenges with other people and win prizes: Social comparison/Non-specific reward/incentives. Moreover, the biofeedback data (represented by the detection and communication of the heartbeat) and the Behavior Goals Review (Michie et al., 2013) will be the core functions of the application.

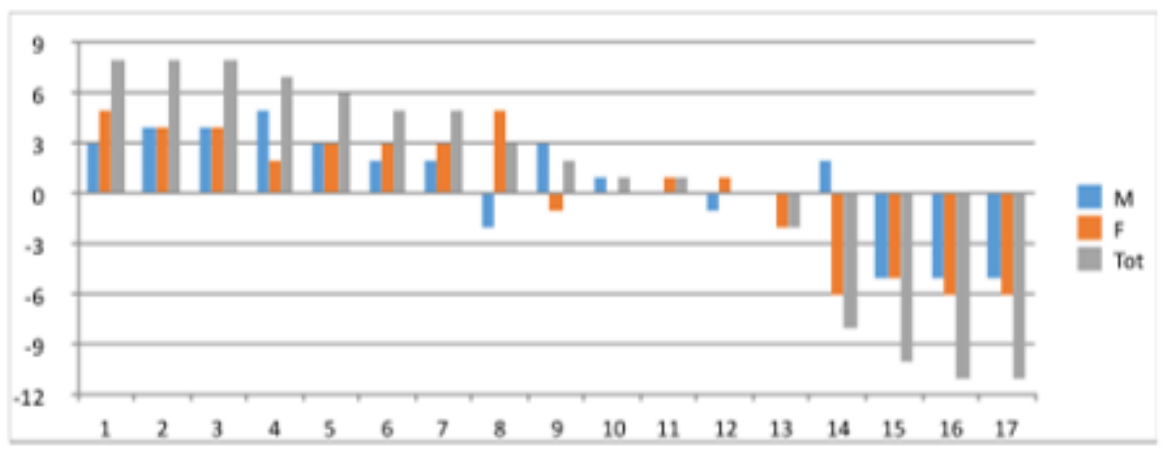

Figure 2. Motivation

\subsection{The User-Test Sessions}

The test was structured in three phases: a warm up interview, the usability test was administered and then an interview to understand the catch some highlights of the overall experience. A heuristic analysis was carried out too. The usability test was conducted during January 2017. The general test aim was to:

- Evaluate the ease of use and comprehensibility of interface, interaction and information architecture.

- Identify the ambiguities and critical issues related to design, IA and the flow of information.

The method chosen for conducting the usability test is the scenario-based, which provides for the definition of use scenarios that show some typical and plausible action contexts and target to be achieved. To identify the number of suitable participants, there was made a reference to the Nielsen theory which considers that with 5 users it is possible to identify $85 \%$ of the system problems. In this way, the first 5 users are able to highlight practically all the problems and the subsequent ones will do nothing but add some element (Nielsen \& Landauer, 1993). The experimental sample was composed by 10 participants (5 men and 5 women), age 30-50 with a average to high technological skills and knowledge. Five of them have already been using or used at least once a wellness or exercise app, the other were newbies to the field. After having explained the test target (that is to verify the usability of the app and not person's capabilities) the tasks were given verbally one at a time and, during the participants' performance, they were observed and recorded. Among the tested, were also enrolled two experienced users, who during the course of the task, have highlighted in depth problems and strengths of the app. During the fulfillment of the task, therefore, users were invited to highlighting doubts and critical issues stimulated by the interaction. This method, called thinking aloud (Nielsen, 1994), allows the facilitators to understand motivations underlying the participants' choices and to highlight the so-called pain points. 


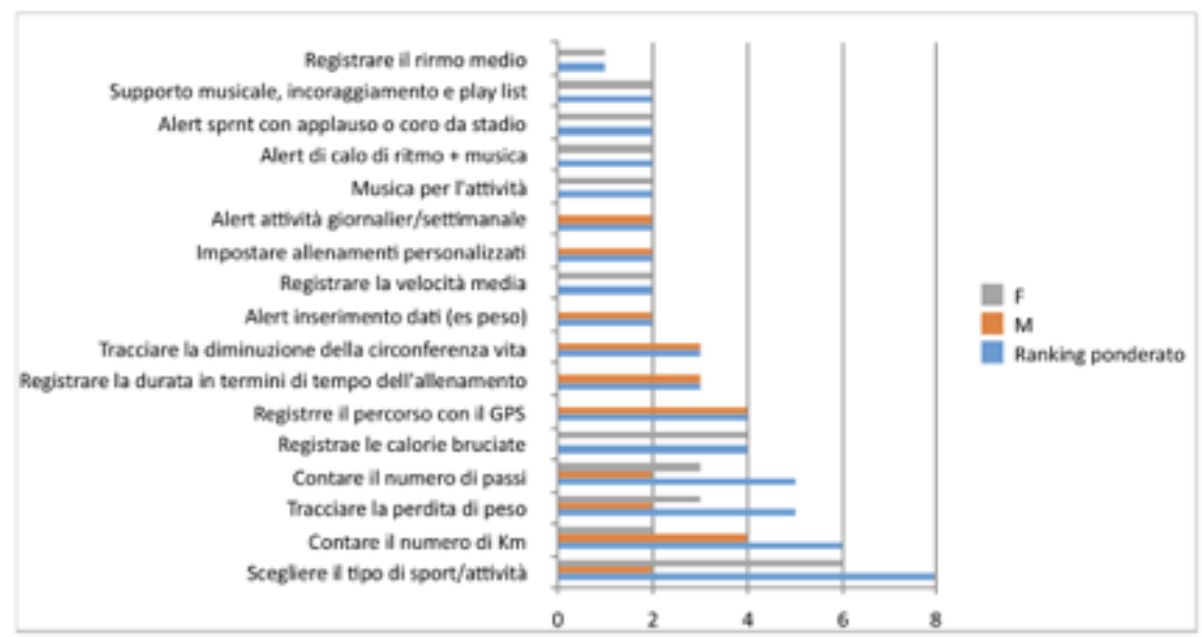

Figure 3. Feed-back and monitoring

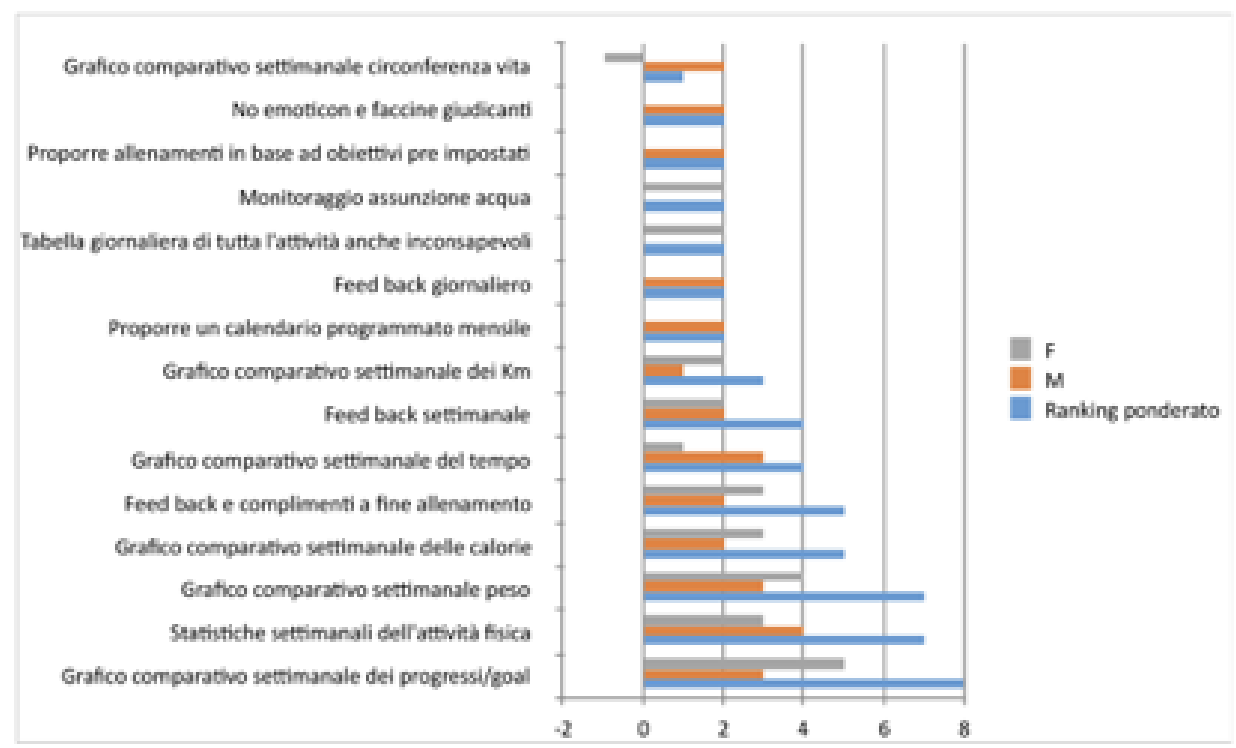

Figure 4. Feed-back and monitoring

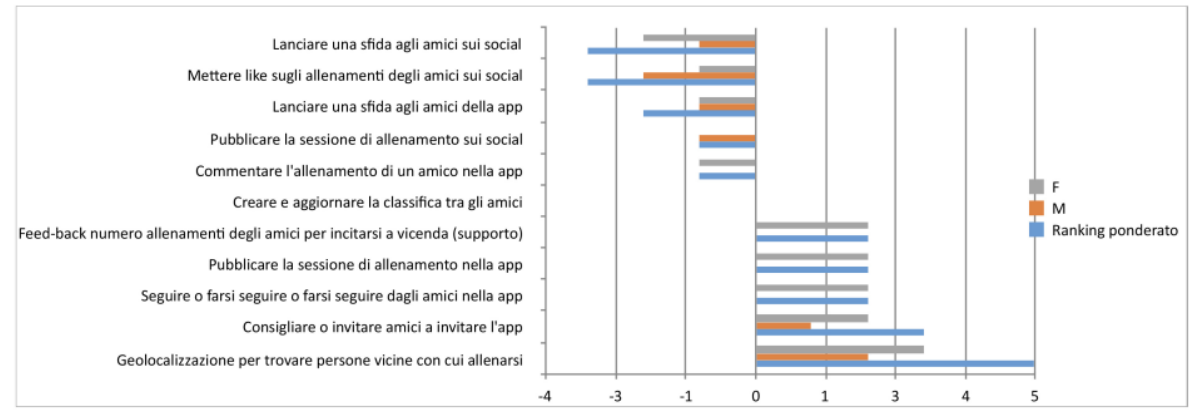

Figure 5. Challenges and Social 


\subsubsection{Scenarios and Tasks}

The subjects were asked to fulfill 9 different tasks corrispondono to the app main o critical features:

1. Registration You have just downloaded Muoviti!, an app that supports physical activity and it is mainly based on the detection of cardiac stress. The offers a training plan so I ask you to take a look at it.

2. First training plan View the training plan and assuming you can not carry out the activity on the scheduled day, try to set it up for Wednesday 5th October

3. Training schedule Choose the activity, set the training time and a reminder (alert).

4. invite friends You don't want to train alone: try to find a friend to to train with and set a meeting point.

5. Start training Choose the task to be performed. Imagine that you started training but, because of the effort, you need to rest. After a short pause, restart.

6. Trying conclusion Conclude the training and see how did it go

7. Friend training You have completed the first week of training. See the new program and take part in a friend's training event.

8. Move with a friend Add Gaia80 as friend and train with her.

9. Weight change Controls the decrease in your weight over the last month.
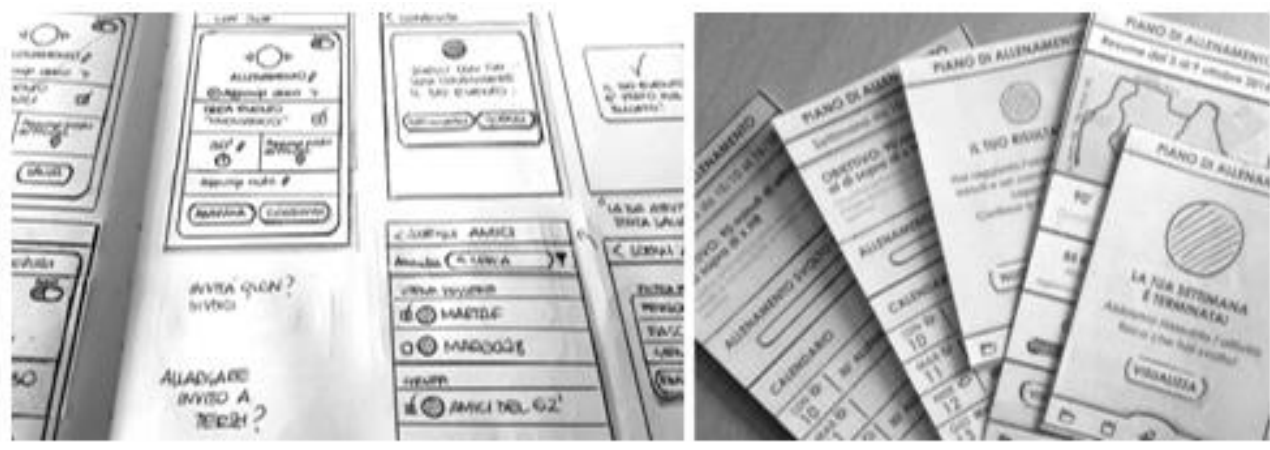

Figure 6. Low-fidelity wireframe and app flow chart (left), paper prototypes used during the user test activities (right)

\subsubsection{User Tests Highlights}

Task 1: registration. No particular problems have been highlighted. One user would prefer that the explanation screens were part of the registration flow forcing the user to read them. The interface used for setting height, waist and weight data were appreciated even if someone suggested, in the weight screen, to arrange the progressive numbering on a half convex upwards circumference in order to remember the one present in the scale. Almost all the participants urged to have the possibility to choose more than one most practised sport. That reveals a misunderstanding because it was suggested to set it by default. Task 2: first training plan. This task was difficult for most of the users who would like to change the training day directly, without the tap of the edit button. Someone suggests to change the text "HR" with "aerobic threshold". To reduce the scrolling, a user advised to use a carousel to see the explanation and the progress bar. One person would like to integrate the app calendar with the one in the smartphone. Furthermore, two users would like to do more training than the scheduled ones.

Task 3: training card. The change of the scheduled training was a bit difficult. They expect to be able to change information directly but almost half of users think they do not need a save button. The possibility of set an alert is considered very useful: some users prefer a default setting, while others would like to have the possibility to change it. One user would like the reminder ringtone to be customizable and another one would like to receive an email as a reminder. To change the sport, users suggest to immediately show four icon of the main sports instead of the gallery to scroll. Regarding the selection of the time, everyone expects that at the click of the word not defined appears a keyboard that allows the insertion of the time or the time picker.

Task 4: invite friends. For creating a training event, users recommended to change the label (share with friends, lookfor friends). A participant would like the "share with friends" button to be visible only after filling out the main board. In particular, users would like to set the meeting using Google Maps and to remember the favorite places. Users don't know what the check for selecting an event as private means. About the friends list, several people would like to select friends among their phone contacts, others would like to find some 
Facebook friends and only one person expects to see friends/Whatsapp contacts. The filters of friends should be reviewed because the age group was not considered useful, while there should be added: main sport, match (to find people with same training habit) and level. A user suggests placing the training level symbol near the person's icon. Several participants would like to be able to create customized groups.

Task 5: start training. Changing the sport selection was difficult: only one user succeeded it. All users expect that when training time is reached, the app registers it and sends an alert to the user (sound or vibration) saving it automatically. Two users suggested to add a confirmation screens when the time is reached in order to let the user continues his training if he/she wants. The icon chosen for the connection with the PulseOn turned out to be completely incomprehensible.

Task 6: training conclusion. Almost all users raised concerns about the scale of self-efficacy: the blue emoticon was considered not appropriate to represent a person with high self-efficacy. It was suggested to use a classic "traffic lights" sequence. Some users have recommended making the heartbeat more important and to show also its graph to visualize any peaks and decreases.

Task 8: move with friends. Regarding the friend's profile, two users thought that the label "shared training" allows the access an area where they were visible shared.

Task 9: weight change. The register area was chosen by exclusion. One user has not completed the task. (For an overall idea of the tests' results see figure 7 and figure 8).
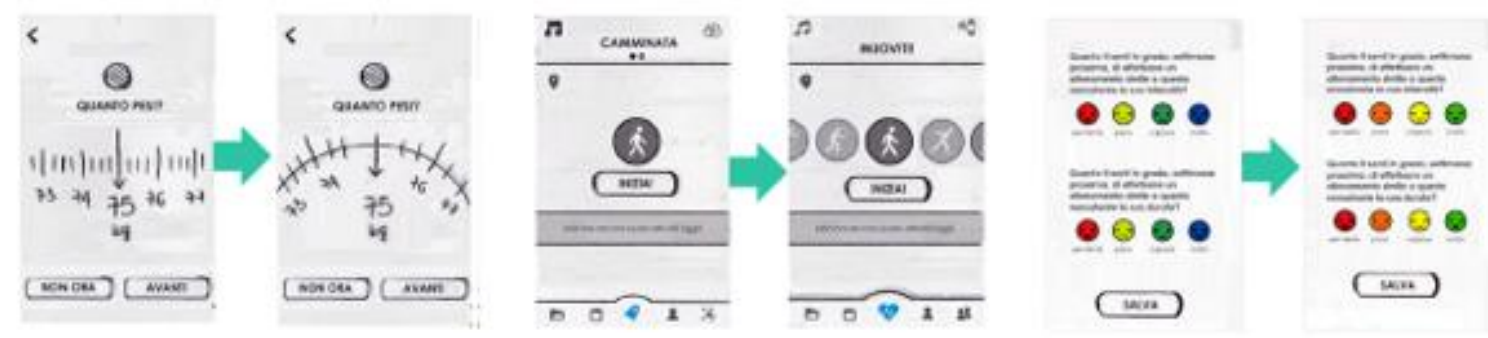

Figure 7. Wireframe redesign iterative cycle: a) settings b) choosing c) assessing

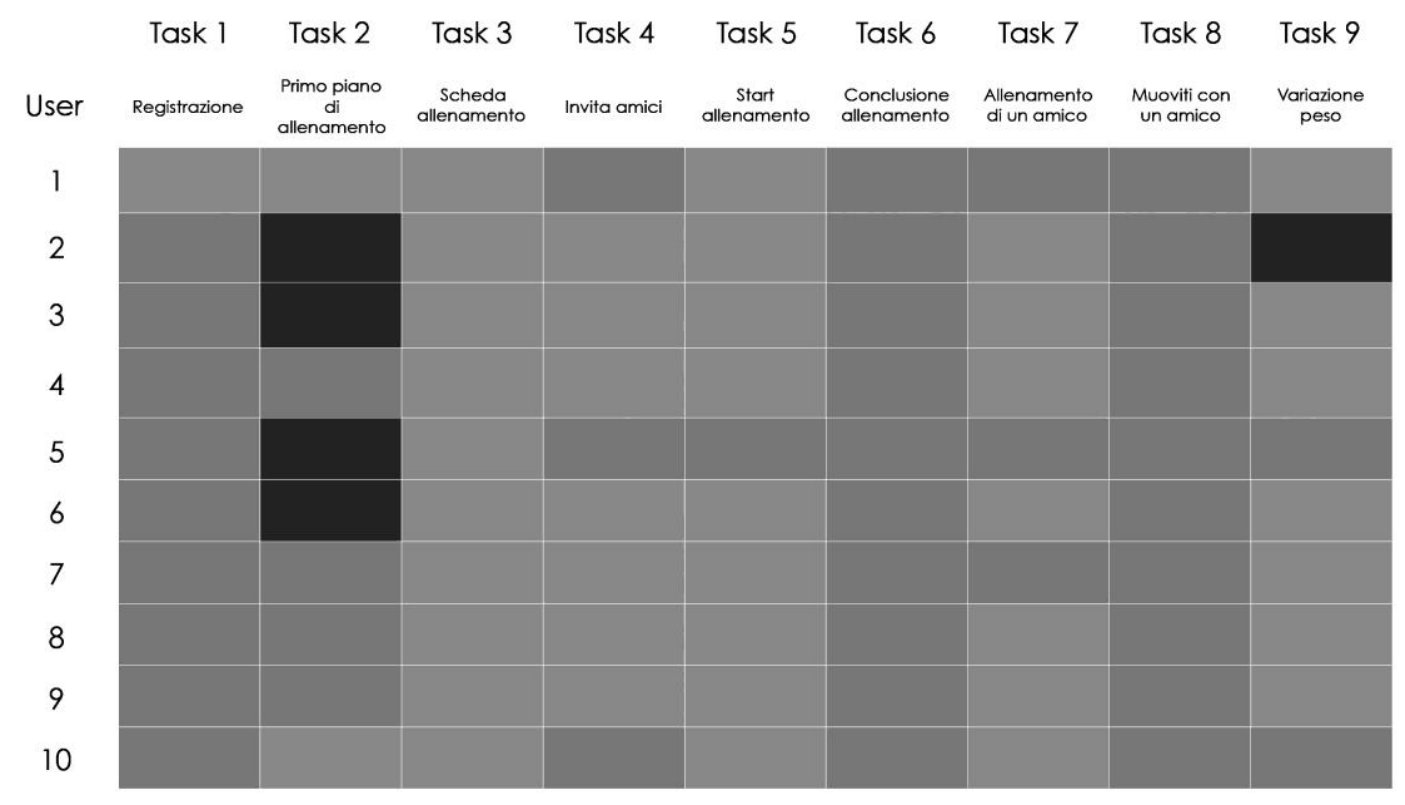

Figure 8. User tests results: Tasks 1-9 / Users 1-10 


\subsubsection{Interviews Highlights}

At the end of the test, users were involved in a brief interview to suggest any improvement and pros and cons encountered using the app. Users evaluated very positively the app ability to create a personalized training and its features. The interfaces developed for setting height, weight and waist data, and the presence of the weather on the training calendar were also highly appreciated.

Among the negative notes were underlined: the little comprehensibility of some icons, the language used in some areas of application (too technical, e.g. HR) and the presence of too many complex steps. Some users believe in the app capacity to increase their training and someone would like to be rewarded with discounts (at a hospital, for later services such as Spotify) when the user respect it. A person suggested the availability of justifications in case of missing workout while another one would like to receive notification in case of that they are sitting too long. A lady suggested to add a diet area. Almost everyone expects Muoviti! to send monthly notifications to remind weight and waist circumference updating. Privacy, data management and safety is really important, especially among women. In particular someone asked for an emergency button to contact 112 (emergency call).

\section{CONCLUSION}

The development of Muoviti! has been made possible thanks to an interdisciplinary team (psychologists, designers, computer scientists, and software developers), respectful of the skills and thoughts to the respective knowledge and skill: the psychological requests have shaped the design and the design, both of the experience, and the interaction/interface - have been trying to listen carefully to the user needs. Furthermore, software developers have been devoting themselves to the structuring of a solid model of data processing supported by the user research.

The experimental work done with the users has allowed identifying not only their needs but also expectations and fears regarding a delicate topic such as one's own health. Among the issues that have emerged and initially underestimated there are mainly to aspects: the need for privacy particularly critical for women for personal security reasons, and an ambivalent relationship with the challenges and competitions.

On one hand, according to some participants' suggestion, asking, for example, for the introduction of the user-level passage and prize achievement (discounts or promotions) by the results achieved, the application would increase its play mechanics. The process, called gamification, includes the application of gaming metaphors in non-game contexts to influence behaviour, improve motivation and enhance engagement. Criticism to the approach underlines that gamification replaces intrinsic motivation with extrinsic motivation, thus pushing a person to engage in an activity not because he finds it stimulating and rewarding, but for the prize that will receive in return by performing a specific action. The intrinsic motivation, on the contrary, derives from internal factors and is realised when a person engages in an activity because he finds it stimulating and gratifying in itself, and feels satisfaction in feeling more and more competent. On the other hand, some participants showed a marked aversion to the challenging issue, focusing instead on a more inclusive and collaborative social participation.

Some critical points too have been identified during the research activities. The first lies in the test procedure. The evaluation of user experience and usability have been assessed through paper prototyping. This approach as well as offering some advantages such as stimulating the imagination and allowing users to focus on the features present, has the limit to convey a different experience compared to that of a real application. In the future, it could be useful to actually use the app and verify the users' appreciation.

A second limit, however, lies in the design of a hybrid application that, therefore, does not have features perfectly adaptable to different devices. The last one is represented by the heart rate monitor itself. PulseOn should be replaced by a waterproof device characterized by a more powerful transmission capacity and an internal memory. In this way, the user would be allowed to practice his business without taking the phone but keep it nearby and/or download the data back home.

Finally, among possible future developments, it would be interesting to examine the gender differences that seem to emerge from this first research project. In fact, the women participating in the co-design and user test activities have shown interest or concern for some specific topics that are otherwise ignored by men. Even if, from the results carried out, it is not possible to say whether these are isolated issues or represent a way of dealing with the problem of sporting activity and more generally of personal health. 


\section{ACKNOWLEDGEMENT}

The authors wish to express their thanks the colleagues Dario Baretta, Andrea Greco, Marco D'addario and Patrizia Steca, Department of Psychology of the University of Milano-Bicocca; Fabio Sartori, Riccardo Melen and Fabio Stella, Disco Dept. of Informatics, Systems and Communication of the University of Milano-Bicocca and all the participants to the experimental phase of the project.

Although the paper is a result of the joint work of all the authors, Letizia Bollini is in particular author of paragraphs 1, 2, 3, 3.1 and 4 and Monica Pennati is author of paragraphs 3.2.

\section{REFERENCES}

Alessandri, N. e Viroli, M., 2007. Wellness. Storia e cultura del vivere bene. Sperling \& Kupfer, Milano.

Ashford, S, Jemma, M., and French, DP, 2010. What is the best way to change self-efficacy to promote lifestyle and recreational physical activity? A systematic review with meta-analysis. British journal of health psychology 15 , No 2, pp .265-288.

Baretta, D., Sartori, F., Greco, A., Melen, R., Stella, F., Bollini, L., \& Steca, P., 2016. Wearable devices and AI techniques integration to promote physical activity. In Proceedings of the 18th International Conference on Human-Computer Interaction with Mobile Devices and Services Adjunct. Firenze, Italy, pp. 1105-1108.

Bauman, A. E, Reis, R. S, Sallis, J. F, Wells, J.C, Loos, R. J. F., \& Martin, B.W., 2012. Correlates of physical activity: Why are some people physically active and others not?, Vol. 380, 258-271.

Bollini, L., 2017. The social representation of a territory. Designing the user experience of spatial interaction from a user-centered theoretical perspective. Pad, No. 13, pp, 170-191.

Bollini, L., De Palma, R., Nota, R. \& Pietra, R., 2014. User experience \& Usability for mobile geo-referenced apps. A case study applied to cultural heritage field. In B. Murgante et al. (Eds.), Computational science computational science and its applications - ICCSA 2014. Part II, LNCS 8580. Springer-Verlag, Berlin Heidelberg, pp. 652-662.

Bollini, L., 2012a. Visual design for non-designers. A participatory experience based on web 2.0 tools in a multidisciplinary master degree programme. In Chova, LG; Martinez, AL; Torres, IC (Eds.) Proceedings Inted2012 conference. IATED, Valencia, pp. 3157-3162.

Bollini, L., 2012b. Comunicare con il colore spazi e percorsi: Aspetti metodologici, ergonomici e user-centered. Campus bicocca: Un caso studio. In M. Rossi \& A. Siniscalco (Eds.), Colore e colorimetria. Contributi multidisciplinari, Vol. VIII. Maggioli Editore, Rimini, pp. 431-438

Burke, V., Beilin, L. J., Cutt, H. E., Mansour, J., \& Mori, T. A., 2008. Moderators and mediators of behaviour change in a lifestyle program for treated hypertensives: a randomized controlled trial (ADAPT). Health education research, Vol. 23, No. 4, pp. 583-591.

Caulfield, B. M., \& Donnelly, S. C., 2013. What is Connected Health and why will it change your practice?. QJM, Vol. 106, No. 8, pp.703-707.

Haskell, W. L., Lee, I-M., Pate, R. R., Powell, K. E., Blair, S. N., Franklin, B. A., \& Bauman, A., 2007. Physical activity and public health: Updated recommendation for adults from the American College of Sports Medicine and the American Heart Association. Medicine and Science in Sports and Exercise, Vol. 39, No. 8, pp. 1423-1434.

Kaewthummanukul, T., \& Brown, K. C., 2006. Determinants of employee participation in physical activity critical review of the literature. AAOHN Journal, Vol. 54, No. 6, pp. 249-261.

Klein, M. C., Manzoor, A., Middelweerd, A., Mollee, J. S., \& te Velde, S. J., 2015. Encouraging physical activity via a personalized mobile system. IEEE Internet Computing, Vol. 19, No. 4, pp. 20-27.

Michie, S., Richardson, M., Johnston, M., Abraham, C., Francis, J., Hardeman, W \& Wood, C. E., 2013. The behavior change technique taxonomy (v1) of 93 hierarchically clustered techniques: building an international consensus for the reporting of behavior change interventions. Annals of behavioral medicine, Vol. 46, No. 1, pp. 81-95.

Nielsen, J. \& Landauer, T. K., 1993. A mathematical model of the finding of usability problems, Proceedings of ACM INTERCHI'93 Conference. Amsterdam, The Netherlands, 24-29 April, pp. 206-213.

Nielsen, J., 1994b. Heuristic evaluation. In Nielsen, J., and Mack, R.L. (Eds.), Usability Inspection Methods. John Wiley $\&$ Sons, New York, NY.

Rizzo, F., 2009. Strategie di co-design. Teorie, metodi e strumenti per progettare con gli utenti. FrancoAngeli, Milano.

Strath, S. J., Kaminsky, L. A., Ainsworth, B. E., Ekelund, U., Freedson, P. S., Gary, R. A., ... \& Swartz, A. M., 2013. Guide to the assessment of physical activity: Clinical and research applications. A scientific statement from the American heart association. Circulation, Vol. 128, No. 20, pp. 2259-2279.

WHO, World Health Organization, 2010. Global recommendations on physical activity for health. http://www.who.int/dietphysicalactivity/publications/9789241599979/en/ [2017, January 10]. 